\title{
Factor Fluctuation in Super Short Avalanche Electron Propagation
}

\author{
Omar Rodríguez Pinilla, Fernando Díaz Ortiz, Carlos Gómez, and Mikel F Hurtado M
}

\begin{abstract}
Compact Intracloud Discharges (CID) and most of Transient Luminous Events (TLE) are two known microsecond-pulse discharges related to electrical activity of thunderclouds. However, their nature and relationship with other cloud discharges still unclear. Few theoretical models had been proposed to explain the nature of this phenomenon. Some proposed models involved the effects of runaway electron avalanches (REA) and relativistic runaway electron avalanches (RREA) as essential part of the aforementioned discharges. In this work, an initial stage is done to propose new models to explain behavior of CID and TLE. Thus, it is simulated the propagation of a charged particle for a short distance, emulating a supershort avalanche electron beam (SAEB). Specifically, first results presented come from simulating the displacement of a charged particle and finding its fluctuation factor by means of perturbations theory. Other works on this issue has been done using different approaches namely, Feynman integrals with similar outcomes. Perturbation theory is used because in order to allow a future interaction in the model among particles, the terms of the perturbation series can be manipulated using Feynman diagrams. Initial conditions assumed for this work are: unit cell, anisotropic volume and $\mathbf{N}$ molecules inside the volume with no interaction. Three relevant conclusions can be exposed from the results, the simulation is coherent with the obtained results using Feynman integrals approach and the equations make possible to predict the amount of photons generated during the avalanche. Finally, it is possible to model charged particle generation through annihilation of variations of electric potential. Future work includes update the model to consider the interaction among molecules and perform experimental validation of the proposed model.
\end{abstract}

Index Terms-Avalanche; Electric Discharge; Fluctuations Factor; Storm Cloud; Integral Roads.

\section{INTRODUCTION}

Taking as approximation a cubic volume of gas (unitary cell) in which several molecules of different chemical configuration can be distinguished, it can be considered amorphous, when the atoms of the volume carry out in addition to thermal oscillations, forced oscillations under the action of an external force, the Hamiltonian of disturbance proper to the volume can be expressed in the following way [1],[2]:

$$
\widehat{H}^{\prime}=\sum_{j, n} \hbar w_{j, n} \widehat{X}^{-1}{ }_{j, n} \widehat{U}_{j, n}
$$

Published on December 20, 2018.

Authors are with the Department of Electronic Engineering, Universidad Central, Bogota, 110311, Colombia. (e-mail: orodriguezp@ucentral.edu.co) where: $\widehat{U} \rightarrow$ shift operator of the equilibrium position of atoms in a randomly chosen molecule of gas; $\hat{X}^{-1} \rightarrow$ correction operator by displacement of the equilibrium position of the atoms in the gas molecule caused by an external force

Replacing: $\widehat{X}^{-1}{ }_{j, n} \widehat{U}_{j, n}=\alpha_{j, n}$ as deformation parameter of the gas molecule, and depending on the configuration by atomic bond, the gas will deform differently for each direction of movement of the beam of charge carriers (beam). In order for the first charge carrier in the beam to begin its movement, the tension in the gas and the electric field formed in the cloud must generate the necessary conditions for the known phenomenon of creation and annihilation of vacancies [3], [4], a process similar to that generated in a semiconductor, then, the Hamiltonian perturbation of a gas molecule when it begins its movement the flow of charge carriers will be:

$$
\widehat{H}_{1}=\sum_{s} \hbar w_{s}\left(a_{s}^{+} a_{s}^{-}+\frac{1}{2}\right)-\sum_{s} \hbar w_{s}\left(a_{s}^{+} s_{s}^{*}+a_{s}^{-} \varsigma_{s}\right)
$$

The SI unit for magnetic field strength $H$ is $\mathrm{A} / \mathrm{m}$. However, if you wish to use units of $\mathrm{T}$, either refer to magnetic flux density $B$ or magnetic field strength symbolized as $\mu_{0} H$. Use the center dot to separate compound units, e.g., "A· $\mathrm{m}^{2}$."

Where: $w_{s}(s=\vec{q}, \sigma) \rightarrow$ normal oscillation frequencies, corresponding to the network $\sigma$ associated with the vector $\mathrm{q}$.

The operator:

$$
S_{s}=-\sum_{j, n, \alpha} K_{j, n}^{\alpha}(t) \frac{1}{w_{s}}\left(\frac{1}{2 \mu \hbar w_{s}}\right)^{1 / 2} V_{j}^{\alpha}(s) \exp (i \vec{q} \vec{n})
$$

where: $K_{j, n}^{\alpha}(t)$ - elastic deformation tensor; $\mu$ - mass of the unit cell; $\vec{q} \vec{n}=\vec{k} \overrightarrow{r_{n}}$ - phase angle of the electronic wave; $\mathrm{V}_{\mathrm{j}}^{\alpha}(\mathrm{s})$ - vibration amplitude of the $\mathrm{j}$ - atom of the zero network; $\alpha=1,2,3$. allows to select an own function of the Hamiltonian (2), such that the eigenvalues of the operator (energy values) are only affected by an energy correction term, to the values of a quantum harmonic oscillator, due to this, a unitary operator can be chosen such that, it fulfills the following condition:

$$
\widehat{U}=\exp \left(\sum_{s}\left(a_{s}^{+} \varsigma_{s}^{*}-a_{s}^{-} \varsigma_{s}\right)\right)
$$

and restricting that $\varsigma_{s}=\varsigma_{-s}$ is symmetric to any canonical transformation and also that it is fulfilled that:

$$
\begin{aligned}
& \widehat{U}^{+} a_{s}^{-} \widehat{U}=a_{s}^{-}+\varsigma_{s}^{*} \\
& \widehat{U}^{+} a_{s}^{+} \widehat{U}=a_{s}^{+}+\varsigma_{s}
\end{aligned}
$$


From the above conditions (3), (4) and (5) we obtain:

$$
\left\{\sum_{s} \hbar w_{s}\left(a_{s}^{+} a_{s}^{-}+\frac{1}{2}\right)+\hbar w_{s}\left|s_{s}\right|^{2}\right\} \varphi=E \varphi
$$

where: $\varphi=\widehat{U}^{+} \phi$ From (6) we can extract the energy correction factor mentioned above and that corresponds to the second term in the left part of the equation. For gas, this represents an increase in vibrational energy due to the external factor, represented by the external force or elastic deformation force, which can be calculated as follows:

$$
\begin{gathered}
S_{s}^{*} S_{s}=\left|S_{s}\right|^{2}=\left|K_{j, n}^{\alpha}(t) \frac{1}{w_{s}}\left(\frac{1}{2 \mu \hbar w_{s}}\right)^{1 / 2} V_{j}^{\alpha}(s)\right|^{2}= \\
\frac{1}{w_{s}{ }^{2}} \frac{1}{2 \mu \hbar w_{s}}\left|K_{j, n}^{\alpha}(t) V_{j}^{\alpha}(s)\right|^{2}=\frac{1}{w_{s}^{2}} \frac{1}{2 \mu \hbar w_{s}}\left|\sum_{n} F_{n}(t, s) \delta_{j, \alpha}\right|^{2}
\end{gathered}
$$

where: $F_{n}(t, s)$ - elastic deformation force; $\delta_{j, \alpha}$ - Kronecker symbol. Including the Hamiltonian deviation of electrons produced by the distribution of atoms in the network:

$$
\widehat{H}_{o}=\frac{2 \pi \hbar^{2}}{m} \sum_{j, n} a_{j, n} \delta\left(\vec{r}-\vec{R}_{j, n}\right)
$$

where: $a_{j, n}$ - Fermí deviation length; m - mass of the electron. Before proceeding to write the full Hamiltonian interaction for the crystal, the action:

$$
\vec{k} \vec{R}_{j, n}=\vec{k} \vec{R}_{j, n}^{o}+\sum_{s}\left(\Lambda_{s, j} a_{s}^{-}+\Lambda_{s, j}^{*} a_{s}^{+}\right)
$$

Determine the shift of the $\mathrm{j}$ - atom in the unitary network, in relation to the established coordinate system.

Where: $\quad \Lambda_{s, j}=\sum_{s}\left(\frac{\hbar}{2 \mu^{N} w_{s}}\right)^{1 / 2} V_{j}^{\alpha}(s) A^{\alpha} \exp (i \vec{q} \vec{n}) ; \quad \mu^{N}$ effective mass of the unit cell or initial volume chosen; at the same time that: $\Lambda_{s, j}=\Lambda_{s, j}^{*} ; A^{\alpha} \rightarrow\left(\frac{1}{m^{2}}\right)$ - parameter of deformation of a face of the unit cell, on which the electromagnetic wave or electronic wave have an effect. Due to the above reason, there must be a term that determines the pressure exerted by the electronic wave upon impacting on the material, this term results from the following operation:

$$
\begin{gathered}
\Lambda_{s, j} S_{s}^{*}=\sum_{j, n, \alpha}(-1)\left(\frac{1}{2 w_{s}^{2} \mu^{N+1 / 2}}\right)\left|V_{j}^{\alpha}(s)\right|^{2} A^{\alpha} K_{j, n}^{* \alpha}(t)= \\
\sum_{j, n, \alpha}(-1)\left(\frac{1}{2 w_{s}^{2} \mu^{N+1 / 2}}\right)\left|V_{j}^{\alpha}(s)\right|^{2} \frac{P_{j, n}^{\alpha *}(t)}{a_{o}}
\end{gathered}
$$

where: $a_{o}$ - network parameter; $P_{j, n}^{\alpha *}(t)$ - pressure tensor of the electromagnetic wave. Now yes: $V_{j}^{\alpha}(s)=V_{j}^{\alpha} \exp \left(i \vec{s} \vec{\rho}^{\prime} !\right)$ - is the structural potential of the network, then, (10) will include a term called structural factor of the network. Calculation presented in graph 1

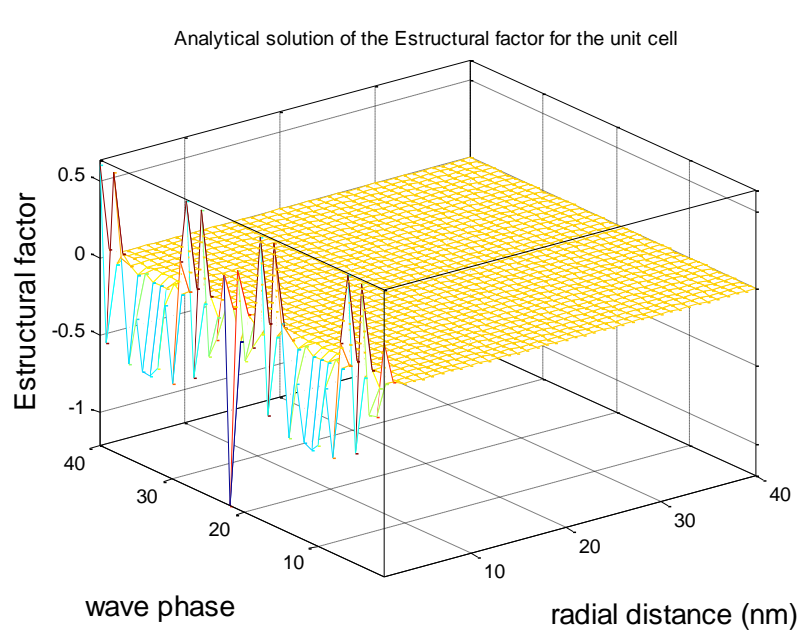

Fig. 1. Behavior of the structural factor of local deformation in an elementary volume of gas

Including the total volume of the gas, the atomic density by volume, the radius of a molecule and the periodic distribution of the action potential, (10) will be:

$\Lambda_{s, j} S_{s}^{*}=$

$\sum_{j, n, \alpha}(-1)\left(\frac{1}{2 w_{s}^{2} \mu^{N+1 / 2}}\right)\left|V_{j}^{\alpha}(s)\right|^{2}|S(s)|^{2} \frac{(2 \pi \hbar)^{3}}{\Delta a_{o}} N_{o} \sum_{\vec{\tau}} \delta(\vec{s}-$

$2 \pi \vec{\tau}) P_{j, n}^{\alpha *}(t)$

where: $\Delta$ - volume of the network; No - atomic density in the gas volume; $S$ (s) - network structural factor.

With the help of the equations associated with the interaction Hamiltonians (1), (2) and (8), we can then write the complete Hamiltonian interaction and also evaluate the propagation of the wave function of electrons in a network unitary, based on the semi classical description of Feynman road integrals [5]-[11].

From (9), the action:

$$
\begin{aligned}
& \Lambda_{s, j} a_{s}^{-}(t)=\sum_{\alpha}\left(\frac{\hbar}{2 \mu^{N} w_{s}}\right)^{1 / 2} V_{j}^{\alpha}(s) A^{\alpha} \exp (i \vec{q} \vec{n})\left[\left(\frac{\mu w_{s}}{2 \hbar}\right)^{1 / 2} \hat{x}+\right. \\
& \left.i \frac{\hat{p}}{\left(2 \hbar \mu w_{s}\right)^{1 / 2}}\right]
\end{aligned}
$$

where: $a_{s}^{-}(t)$ - vacancy creation operator. Fig. 2 shows both the behavior of the vacancy distribution in a unit of gas volume (blue line), and the behavior of temperature (green line). 


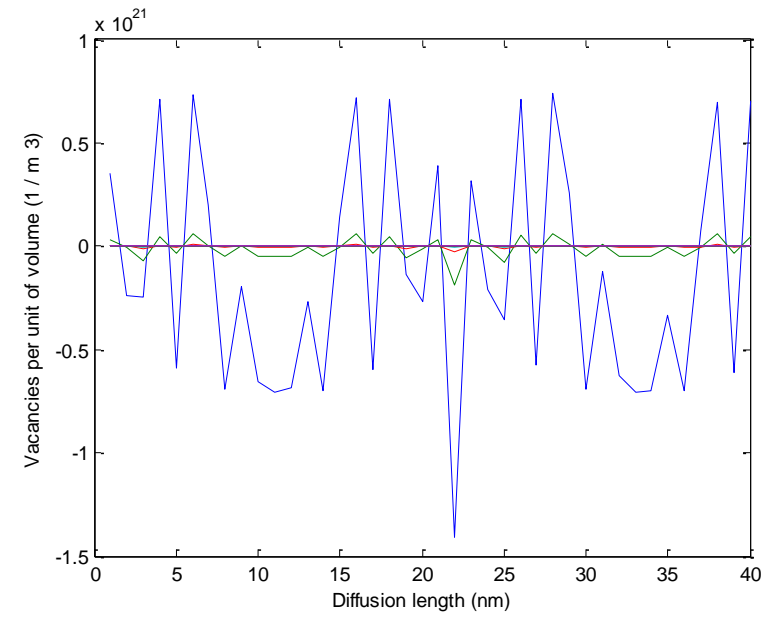

Fig. 2. Simulated behavior of the number of vacancies per unit of volume (blue line) (Equation (12)) and temperature (green line), while the red line represents the factor of fluctuations of the trajectories of the load carriers in the same unit of volume

After the above specifications, the complete Hamiltonian beam interaction with the unit network will be:

$$
H=H_{o}+H_{1}+H^{!}+\left(\frac{\hat{p}}{2 m}+\hat{V}(x)\right)
$$

where: $\mathrm{V}(\mathrm{x})$ - periodic potential of the gas. When the Hamiltonian of interaction does not have the form $\widehat{H}=\widehat{T}+$ $\widehat{V}(x)$, then the propagator or function of Green has a more general form:

$$
G\left(x, t ; x_{o}, t_{o}\right)=N \int_{-\infty}^{\infty} \vec{D}_{x} \exp \left\{\frac{i}{\hbar} \int_{t_{1}}^{t_{2}} L(x) d t\right\}
$$

With: $N=\lim _{n \rightarrow \infty}\left(\frac{m}{i h \tau}\right)^{n / 2}$ - factor of fluctuation of the particle of its semiclassical trajectories; $\vec{D}_{x}=\prod_{j=1}^{n-1} d \vec{x}_{j}$ Lagrangian of the particle.

In the semi classical approach:

$$
\frac{i}{\hbar} \int_{t_{1}}^{t_{2}} L(x) d t \cong \frac{i}{\hbar} \tau \sum_{j=0}^{n-1}\left(P_{j} \dot{q}_{J}-\widehat{H}\right)
$$

where: $P_{j}$ - j impulse and $\dot{q}_{j}-\mathrm{j}$ generalized velocity of the $\mathrm{j}$ particle.

\section{COMPUTATIONAL MODEL}

The next and last step of the present work consisted in simulating the fluctuation factor of the possible trajectories of the load carriers in the hypothetical volume established at the beginning of the research work, which can be shown in Fig. 1, likewise the number of vacancies per unit of volume (Equation (12)) depending on the temperature of the discharge Fig. 2. An important part and fundamental contribution of the present theoretical research work was the fact of being able to calculate and simulate the fluctuation factor that follows the flow of load in the storm cloud and one of the possible trajectories of the load carriers in the established volume, as a boundary condition, at the beginning of the document. (Fig. 3 and 4 respectively).

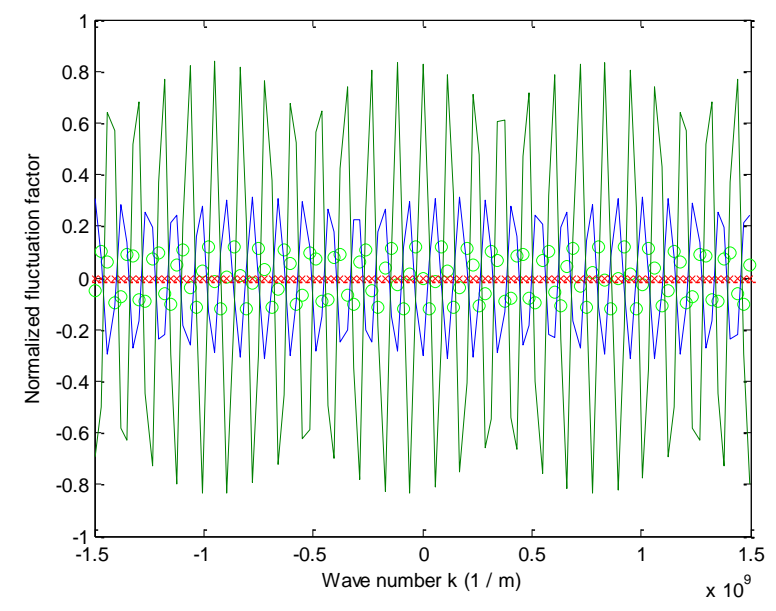

Fig. 3. Simulated distribution of the factor of fluctuations of the trajectories (Equation (14)) of the load carriers (blue line), in the established volume.

The red lines and green points at the origin of the graph represent the temperature variations, while the continuous green lines represent the carrier wave of the magnetic field generated by the current in the discharge

Another of the interesting simulations developed in this report, was to find the trajectories at the beginning of the discharge in the storm cloud, before the onset of the avalanche, this can be seen in Fig. 4.

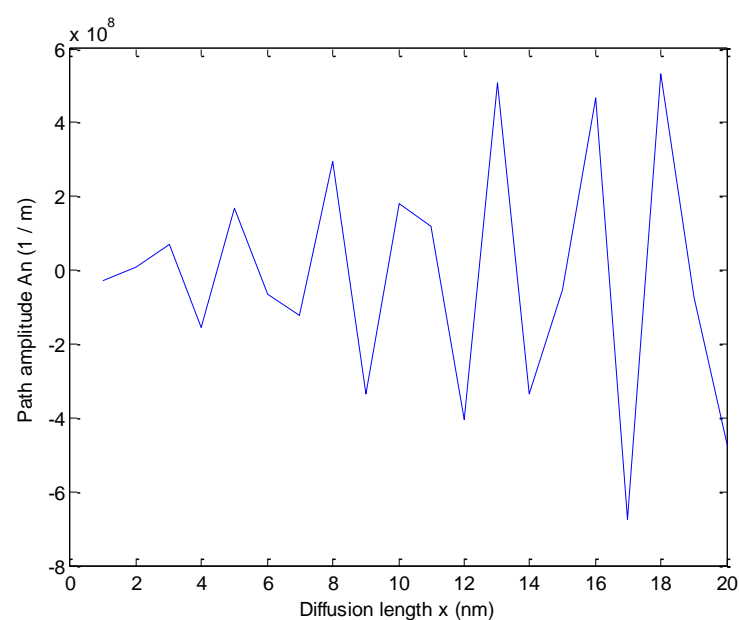

Fig. 4. Simulation of the possible trajectories followed by a load carrier in the storm cloud (Equation (15)), before the onset of the avalanche in the storm cloud

\section{CONCLUSIONS}

In the present theoretical development, supported by computational models of simulation, it can be inferred that the form of the distribution of vacancies in the volume (in a hypothetical unitary cell), determines the path or trajectory that a load carrier can follow in the storm cloud under conditions of energy fluctuations or second quantization.

\section{REFERENCES}

[1] J. D. Bulnes. Propagadores cuánticos calculados de acuerdo con el postulado de Feynman con caminos aproximados por polinomios. Revista mexicana de física e 55 (1) 34-43. (2009).

[2] Devendraa Siingh. Et al. Journal of Atmospheric and Solar-Terrestrial Physics. Volume 134, November 2015, Pages 78-10.

[3] Victor F. Tarasenko. Et al. Review of supershort avalanche electron beam during nanosecond-pulse discharges in some gases. Matter and Radiation at Extremes 2 (2017) 105e116. 
[4] B J Berne, and D Thirumalai. On the Simulation of Quantum Systems: Path Integral Methods. Annual Review of Physical Chemistry. Vol. 37: 401-424 (Volume publication date October 1986).

[5] T. Czech, A. T. Sobczyk, A. Jaworek, et al. Journal of Electrostatics. Volume 70, Issue 3, June 2012, Pages 269-284.

[6] Earle R. Williams. Electricity in the Atmosphere: Global Electrical Circuit. Encyclopedia of Atmospheric Sciences (Second Edition), 2015

[7] Fabio Nicola. Convergence in $L^{p}$ for Feynman path integrals. Advances in Mathematics. Volume 294, 14 May 2016, Pages 384 409.

[8] S. Albeverio and S. Mazzucchi. The time-dependent quartic oscillator - a Feynman path integral approach. Journal of Functional Analysis 238 (2006) 471-488.

[9] Naoto Kumano-go. Phase space Feynman path integrals with smooth functional derivatives by time slicing approximation. Bull. Sci. math. 135 (2011) 936-987.

[10] Preben Hvelplund. Et al. Experimental studies of the formation of cluster ions formed by corona discharge in an atmosphere containing $\mathrm{SO}_{2}, \mathrm{NH}_{3}$, and $\mathrm{H}_{2} \mathrm{O}$. International Journal of Mass Spectrometry. Volumes 341-342, 1 May 2013, Pages 1-6.

[11] Rodríguez P. Omar. Uv radiation by the Debye sphere interaction plasma - metal nanoparticles on the surface of plant tissue. International Journal of Applied Engineering Research and Development (IJAERD) ISSN(P): 2250-1584; ISSN(E): 2278-9383. Vol. 7, Issue 3, Jun 2017, 11-16.

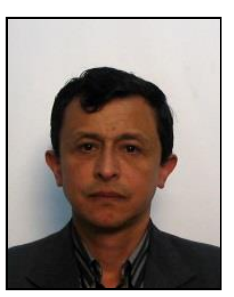

Omar Rodríguez Pinilla. MsC in nuclear physics. Author of more than 25 published in indexed journals. Director of the Maxwell research group attached to the Central University.

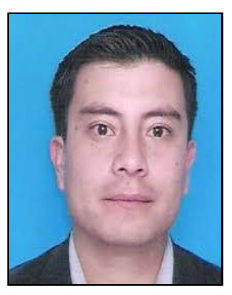

Fernando Díaz Ortiz. Fernando Diaz-Ortiz received the B.S degree in electronic engineering and the M.S. degree in information science and communications from Universidad Distrital Francisco Jose de Caldas, Bogota, Colombia in 2001 and 2015. He received the M.S. degree in electronic engineering from Pontificia Universidad Javeriana, Bogota, Colombia in 2011. $\mathrm{He}$ is currently working toward the $\mathrm{PhD}$ degree in Universidad Nacional de Colombia. He is currently Assistant Professor in the Department of Electronic Engineering, Universidad Central, Bogota, Colombia.

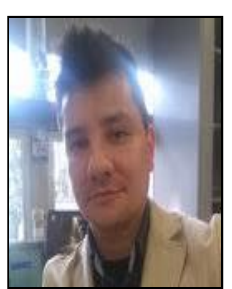

Mikel Fernando Hurtado M- Chemist, Ms.c Chemical Thermodynamics, MsC, Materials Science and Nanotechnology, PhD, Solid State Chemistry Associate Professor.

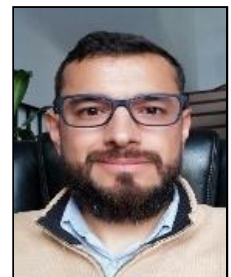

Carlo A. Gómez V. He was born in Bogotá Colombia. He received his B.Sc in Electrica Engineering of the Universidad Nacional de Colombia in 2004. He has been a professor of the Universidad Central de Colombia since 2006, and is author of three invention patents. He has also worked as a researcher in the Electromagnetic Compatibility Group of the Universidad Nacional de Colombia (EMC-UNC) and in the MAXWELL research group of the Universidad Central, in areas such as high voltage, electromagnetic compatibility and gas discharge. He currently studying his $\mathrm{PhD}$ in the gas discharge area. 